\title{
La importancia de los espacios y escenarios deportivos para la práctica de la recreación y el deporte
}

\author{
The importance of sports spaces and stages \\ for the practice of recreation and sports.
}

José Nodier Liévano Ortiz ${ }^{1}$

Secretaría de educación de Bogotá

Recibido: 21.07.2021

Aceptado: 30.09 .2021

\section{Resumen}

El presente artículo hace parte de los resultados de la investigación: "La importancia de los espacios recreo deportivos para la práctica pedagógica de actividad física en el colegio distrital Carlos Albán Holguín”. Este proceso se inicia desde al año 2007 y finaliza en el año 2019. El proyecto surge al observar la importancia y percibir el estado de deterioro en que se encuentran los espacios y escenarios para la práctica de las actividades recreativas y deportivas en el colegio Distrital Carlos Albán Holguín, perjudicando a los estudiantes y docentes de la educación básica secundaria. La presente es una investigación de corte cualitativo-basada en un diseño desde la investigación acción educativa, cuyo objetivo principal es generar reflexión a partir de las realidades educativas existentes; en este sentido se utiliza como técnica las encuestas realizadas a la comunidad educativa de la institución, para develar estas realidades. Los resultados presentados en la investigación demuestran la necesidad de tener varios

\footnotetext{
1 jlievanoo@gmail.com https://orcid.org/0000-0003-3443-6040
} 
espacios, con buenos escenarios y elementos de calidad para la práctica de actividades recreativas y deportivas a desarrollar en el proyecto y en las clases de educación física de acuerdo con lo propuesto por la comunidad educativa.

Palabras Clave: Espacios, escenarios, clases, práctica, Educación Física, deportes, vida saludable, aprovechamiento del tiempo libre, recreación, implementos.

\begin{abstract}
This article is part of the research results: "The importance of sports recreation spaces for the pedagogical practice of physical activity in the Carlos Albán Holguín district school". This process begins in 2007 and ends in 2019. The project arises from observing the importance and perceiving the state of deterioration in which the spaces and scenarios are located for the practice of recreational and sports activities in the Carlos District school Albán Holguín, harming students and teachers of basic secondary education. This is a qualitative researchbased on a design from educational action research, whose main objective is to generate reflection from the existing educational realities; In this sense, the surveys carried out to the educational community of the institution are used as a technique to reveal these realities. The results presented in the research demonstrate the need to have several spaces, with good settings and quality elements for the practice of recreational and sports activities to be developed in the project and in physical education classes in accordance with what is proposed by the educational community.
\end{abstract}

Keywords: Spaces, settings, classes, practice, Physical Education, sports, healthy life, use of free time, recreation, implements. 


\section{Introducción}

Dado que el tema central de este trabajo estará puesto sobre la importancia que tienen los espacios y escenarios deportivos para la práctica de la recreación y el deporte es necesario plantear, algunos parámetros que sirvan de ejes conceptuales. En primer lugar, es preciso entender el concepto de espacios y escenarios deportivos, que son aquellos escenarios adecuados y con buena seguridad donde los docentes de educación física realizan sus prácticas pedagógicas de actividad física, de acuerdo con (Galvis, 2014).

En segundo lugar, desde esta perspectiva, es necesario considerar algunas normas establecidas para la construcción, mantenimiento y los materiales a utilizar en cada una de las actividades programadas por los docentes de educación física, así mismo es importante que se tenga en cuenta en los colegios distritales de la ciudad de Bogotá D.C, especialmente en el colegio Carlos Albán Holguín (CAH), para poder renovar los espacios y por ende las practicas recreativas y deportivas. En tercer lugar, habría que decir también, lo que se pretende es optimizar la calidad y cantidad de los espacios y escenarios, lo mismo que tener los elementos aptos y suficientes para poder así incentivar a los estudiantes a que practiquen actividades recreativas y deportivas. Así mismo, cumplir con la calidad de educación que se debe impartir en las instituciones públicas del país (Pérez, 2016).

Por otra parte, los estudios realizados por Nuviala, Ruiz, \& García, (2003), sobre el tiempo libre, el ocio y actividad física en adolescentes, demuestran que los estudiantes no realizan actividad física y prácticas recreo deportivas a muy temprana edad, los autores explican en su artículo lo siguiente: "la experiencia y el sentido común nos dice que existe un amplio porcentaje de alumnado que no realiza suficiente actividad física ni para mejorar o mantener su salud, ni para consolidar o crear hábitos de práctica física” (p.14).

Esta particularidad puede generar desmotivación ya que los pocos espacios existentes se utilizan en los deportes básicos que se realizan en el colegio CAH (baloncesto, futbol cinco, voleibol) y no se tienen los espacios y escenarios adecuados para la práctica de nuevas 
actividades deportivas-recreativas que puedan impactar a los estudiantes. Refuerza lo anterior el artículo de Pérez Ángel (2016), Educación Física: derecho a la educación de calidad y bienestar donde el autor escribe: "Por otra parte la mayoría de las construcciones escolares oficiales, no cuentan con los espacios y escenarios adecuados, para que los profesores de educación física puedan realizar su trabajo" (p.2). En este sentido, esta situación puede considerarse como un factor asociado, que afecta la motivación frente a la práctica de la recreación y el deporte.

Algo semejante ocurre con las prácticas deportivas y recreativas, referente a la motivación por parte de los jóvenes al realizar ejercicio ya sea en la institución o en su tiempo libre; por tal motivo en el artículo de Mollá (2007), explica lo siguiente: “se presentan en la adolescencia y la juventud, las principales causas por las cuales los estudiantes abandonan la práctica de la actividad física y los motivos que conducen al abandono temporal o permanente de la práctica en los jóvenes están relacionados mayoritariamente con la falta de tiempo libre, los estudios y el aburrimiento" (p.243); lo mismo que la exigencia de las actividades escolares, los juegos y aparatos electrónicos, el cuidar los hermanos menores o estar al mando de los oficios del hogar, todas estas situaciones alejan a los estudiantes, de las prácticas recreodeportivas, aumentando así los índices de sedentarismo.

Así mismo, complementando los artículos de los autores descritos anteriormente, en el ámbito internacional con el tema de los espacios y escenarios recreo deportivos, encontramos otros conceptos que ayudan a la necesidad de los centros educativos de tener buenos espacios para la práctica recreativa y deportiva, por tal motivo según Burillo, Felipe, Gallardo, García, \& Gil ( 2010):

Los espacios deportivos son el marco idóneo donde los alumnos deben adquirir los hábitos de actividad física deportiva en un ambiente donde se potencien las relaciones socioafectivas, sin embargo, el profesorado de esta área educativa se encuentra con múltiples carencias y deficiencias en cuanto a las instalaciones y espacios deportivos adecuados. (p.288) 
Con la definición anterior, los ambientes propicios son aquellos donde existen escenarios seguros adecuados y con la suficiente cantidad de elementos, para el desarrollo de la práctica recreativa y deportiva, se debe agregar que con referencia a la potenciación pedagógica, los buenos espacios, escenarios y materiales pueden incentivar a los estudiantes y profesores a realizar diferentes labores, donde todos participen con entusiasmo, implementando así otras acciones no solo físicas sino recreo deportivas que estimulen el interés general. Por otro lado, al mejorar los espacios, escenarios y el material, la seguridad y variedad de movimientos, se puede lograr un marco idóneo para la práctica y participación masiva de un buen número de estudiantes en estas tareas.

Algo semejante ocurre con el artículo de Tercedor, Jiménez, \& Moya (1994), el cual aclara que, "Cuando se trata de fomentar la actividad física orientada hacia la salud, quizá el conocer las posibilidades y calidad de la instalación de que disponemos sea una de las primeras cuestiones a responder" (p.1). De acuerdo con lo anterior la importancia de tener buenos espacios y escenarios es primordial, para así mejorar las prácticas de recreación y los deportes.

Otro concepto acerca de la calidad, cantidad de los espacios, escenarios y equipamientos en los colegios, es el artículo de Latorre (2008), donde el autor plantea que: "Hoy día, las instalaciones y espacios deportivos de los centros escolares siguen siendo insuficientes y, en muchos casos, mal estructurados e incumplen cuestiones de normativas básicas, lo que les confiere matices de peligrosidad" (p.62).

Por tal motivo, es necesario considerar la calidad de los espacios recreo deportivos, lo mismo que el equipamiento de implementos adecuados para motivar a los estudiantes a realizar la práctica recreativa y deportiva, para así minimizar las posibilidades que estos factores inadecuados afecten las clases.

Así mismo a nivel latinoamericano las dificultades que se presentan en los centros escolares por falta de buenos espacios recreo-deportivos, han llevado a varios autores a investigar sobre 
esta problemática que concierne a la educación, encontramos varios artículos sobre el tema entre ellos el de Benítez \& Sequera (2009) donde el escritor indica que:

Los espacios para la actividad físico-deportiva son de suma importancia para la población venezolana y mundial, pues permite que el individuo tenga la oportunidad de realizar ejercicio físico o deporte que beneficia integralmente a la ciudadanía, evitando así todas las enfermedades no transmisibles como: diabetes, hipertensión arterial, obesidad, entre otras. Además de mejorar la calidad de vida del practicante. (p.1)

Analizando la cita anterior, es importante manifestar que la oportunidad de realizar actividades recreativas y deportivas, en niños y adolescentes puede generar una serie de beneficios en todo su desarrollo integral, previniendo una serie de enfermedades especialmente cardiovasculares y además favoreciendo las interacciones sociales. Los beneficios integrales de la buena práctica de actividad física son varias, a continuación enunciaremos algunas de ellas: buen rendimiento académico, confianza en sí mismo para desarrollar cualquier actividad, estabilidad emocional, mejora la memoria y percibe mejor las situaciones que se le presenten. Algo semejante ocurre con la calidad de vida, ya que esta se ve favorecida al realizar actividad recreo deportiva, mejorando los problemas de stress que afectan a buena parte de la población no solo la estudiantil sino también la de los adultos.

Se debe tener en cuenta con este artículo, que la falta de buenos espacios y escenarios para la práctica de actividad física es un problema a nivel global que preocupa a los Estados, es importante su mejoramiento, su calidad y su seguridad para el desarrollo de la recreación y los deportes.

En ese orden de ideas, a nivel nacional, encontramos el artículo de Pérez (2016), donde el autor manifiesta:

Acá no puede existir dudas por parte de los hacedores de política pública educativa, la recreación, el deporte y la actividad física en la escuela es parte integral de la formación 
para la vida del ser humano, su bienestar, felicidad y permanencia en el sistema educativo. (p.2)

En otras palabras, la construcción de buenos escenarios puede mejorar la calidad de vida y de educación que se necesita, para que nuestros estudiantes permanezcan en el sistema educativo, para conservar y mejorar la salud buscando su bienestar. Por otra parte, los espacios recreo deportivos motivan anímicamente las prácticas saludables, brindando las condiciones que permitan usos adecuados para lo que fueron construidos, “además deben ser ambientes seguros y carentes de amenazas, que sean estimulantes, propicios e inclusivos" (Unesco, 2015, p. 9).

En relación con la institución a evaluar, el colegio Carlos Albán Holguín tiene un área total de 495.05 metros cuadrados y los espacios recreo deportivos son 252.60 metros cuadrados, de los cuales se encuentran en mal estado 134.10 metros cuadrados (huecos, piedras en todo el espacio que esta deteriorado, postes de la luz mal ubicados, un tanque de agua natural a un costado lateral de estos espacios, borde de anden en las canchas múltiples), afectando a la población estudiantil por el peligro que conlleva a utilizar estos espacios en el desarrollo de las actividades recreativas y deportivas durante las clases.

Por tal motivo, si todos los espacios con los que cuenta el colegio se encuentran en buen estado, se logrará involucrar a más personas de la comunidad educativa a que practique ya sea actividades recreativas o realice algún deporte; para el mejoramiento de la salud, la integración, y la calidad de vida. Otra forma de contribuir a que se mejoren los espacios y escenarios recreo deportivos, es la importancia que tienen las actividades deportivas en el colegio $\mathrm{CAH}$, donde participan los estudiantes, para cumplir con el proyecto de ley aprovechamiento del tiempo libre, estas actividades son: fútbol cinco donde hay 38 equipos masculinos y 14 femeninos estando inscritos 406 estudiantes en las planillas de juego, repartidos en cinco categorías tres masculinas y dos femeninas; en baloncesto 19 equipos, en voleibol 19 equipos con 280 estudiantes inscritos en los dos deportes, los dos géneros y distribuidos en cinco categorías. 
Representando aproximadamente un $43.59 \%$ de los 1.101 estudiantes que tiene la sección bachillerato. Estas actividades deportivas se desarrollan durante el año escolar, en horas de descanso, sábados, festivos y dos días de los periodos de vacaciones, que hay durante el transcurso del año lectivo, y cuentan con el apoyo del consejo directivo, rectoría y el consentimiento de los padres de familia.

Según la investigación concluida al mejorar los espacios recreo deportivos, para las prácticas pedagógicas de actividad física, que se encuentran en regular y mal estado (huecos, piedras, postes de la luz, tanque de agua y bordes de anden), no solo en su estructura física, sino también en el número de escenarios existentes en el colegio distrital $\mathrm{CAH}$, se pueden optimizar como apoyo a las prácticas pedagógicas de actividad física y recreo deportiva que se realizan en la institución.

\section{Metodología}

\subsection{Tipo y diseño de la investigación}

Con respecto al problema planteado se contribuye a desarrollar conocimiento ya que dada la importancia del mismo, puede generar tanto impacto que tocara a las puertas de entidades gubernamentales para que se considere mejorar los escenarios deportivos para la práctica de actividad física y deporte; el deseo es tratar de crear conciencia en las directivas de colegios, universidades, secretarías de educación y otros estamentos para que el tema de los escenarios deportivos en las instituciones educativas y las ciudades, sea tenido en cuenta para realizar adecuadamente la práctica deportiva y la actividad física, para mejorar así los hábitos y la calidad de vida en los escolares de todos los centros educativos públicos y privados del país. La investigación desarrollada es de corte cualitativo describiendo los sucesos complejos en su medio natural, porque se desarrolló en un contexto natural tal y como sucede en el espacio del patio para la actividad física de un colegio público de la localidad de Bosa en Bogotá D.C; mediante la observación directa que responde a la naturaleza de la investigación, utilizando las siguientes técnicas de recolección de los datos solicitados: la observación cualitativa u 
observación de campo donde directamente se explora los ambientes, se describen las comunidades, para así identificar el problema motivo de la investigación, las encuestas en sus dos modalidades, abiertas para que los integrantes de la comunidad educativa respondan con libertad en forma clara su percepción sobre el problema presentado y para que se pueda profundizar sobre la respuesta dada, con base a estas respuestas de las preguntas abiertas se hará el cuestionario de las preguntas cerradas, permitiendo un mayor control de los resultados.

Por otra parte, este proyecto se abordó desde la investigación-acción siendo un tipo de investigación aplicada y destinada a encontrar soluciones a dificultades que tenga un grupo, una comunidad, una organización, donde el grupo afectado participa de la investigación, intentando recopilar información a partir de la observación (diario de campo), con encuestas cerradas a estudiantes, directivos, docentes, personal administrativo, de servicios generales y padres de familia, para posteriormente generar teoría a partir de los datos recopilados. En cuanto al paradigma que se utilizo es el construccionismo, el cual se concibe como una teoría sociológica y psicológica del conocimiento, considerando los fenómenos sociales como ciertos, que se desarrollan particularmente en contextos sociales.

Por consiguiente, este proyecto evalúa la percepción en torno a los espacios recreo deportivos, que tiene la comunidad educativa en general del colegio Carlos Albán Holguín, sede A, jornada tarde de la localidad de Bosa al suroccidente de la ciudad de Bogotá D.C, a nivel de estudiantes se analizó con los grados novenos, docentes de diferentes asignaturas, los padres de familia o acudientes son de los mismos estudiantes de grado noveno, los directivos docentes representados por los coordinadores, administrativos y personal de servicios generales para realizar una muestra probabilística.

\subsection{Población}

Esta investigación se lleva a cabo en el colegio Distrital Carlos Albán Holguín de la localidad de Bosa en Bogotá D.C, localizada al sur occidente de la ciudad, limitando al norte con la localidad de Kennedy, al sur con la Autopista Sur hasta la calle 77 sur, calle $60^{\text {a }}$ del municipio 
de Soacha (Cundinamarca) y calle 57R sur con Ciudad Bolívar, al este río Tunjuelito y con la localidad de Kennedy, al oeste río Bogotá y con los municipios de Soacha (corregimiento 1 y comuna 3 la Despensa) y Mosquera (Cundinamarca). El colegio Carlos Albán Holguín, es una de las 27 instituciones públicas con las que cuenta la localidad de Bosa. Es una institución de carácter mixto, tiene tres sedes y dos jornadas, atendiendo los diferentes niveles educativos, desde la primera infancia, hasta la media vocacional. En el año 2017, el colegio cuenta con 5344 estudiantes, 241 docentes, 10 administrativos, 8 directivos docentes y 28 personas de servicios generales, para la prestación del servicio educativo.

Indico así mismo que la población de esta localidad es heterogénea, en su mayoría conformada por personas de extracción campesina, como consecuencia de la migración interna en el país, o ubicada en este sector de la ciudad, debido a la búsqueda de vivienda y subsistencia. En consecuencia, un alto porcentaje de pobladores son desplazados y se ubican en la estratificación 0,1 y 2 del SISBEN, con predomino de familias con mujeres como cabeza de hogar, que devengan su salario del sector productivo informal o de su desempeño como obreras en el sector productivo formal. Por tal motivo para mejorar la calidad de vida de nuestros estudiantes, es necesario que el colegio recupere los espacios existentes en mal estado, para que sean utilizados en la práctica de actividad física, deportes, recreación y en el aprovechamiento del tiempo libre, (Colegio Carlos Albán Holguín, 2018, p.10).

La Sede A del centro educativo, es aquí donde se va a llevar acabo la presente investigación está ubicada en la Calle 72 B N 84-22 Sur entre los barrios Naranjos y Carlos Albán y se conocía como la Escuela Distrital Carlos Albán Holguín, cuenta con amplias instalaciones para la práctica de actividad física pero como se ha descrito existe un buen espacio en mal estado, esperando el proceso de remodelación ya que el colegio se encuentra en lista de espera para que se realicen los trabajos de la construcción anhelada por la comunidad.

Igualmente, en el contexto del colegio expuesto, se aplica el instrumento de recolección de la información a 25 estudiantes de los cinco grados novenos, 5 por cada curso, teniendo en cuenta que 16 son hombres entre los 13 y 16 años y 9 son mujeres entre los 14 y 16 años. Para el 
proceso de la encuesta a estudiantes se solicitó consentimiento informado a los padres o acudientes. Por otra parte, también se encuestaron a diez docentes, uno por cada asignatura del conocimiento, veinticinco padres o acudientes de los mismos estudiantes seleccionados de los grados novenos, tres directivos docentes y dos personas de la parte administrativa y cinco del personal de servicios generales.

\subsection{Técnicas e instrumentos de recolección de datos}

Por ser una investigación de tipo cualitativo, que busca analizar el estado, la cantidad de los espacios y los buenos escenarios para la práctica pedagógica de actividad física, deportiva y el aprovechamiento del tiempo libre, se utilizará la técnica de observación directa, lo mismo que una encuesta de percepción a la comunidad educativa involucrada en la investigación (Hernández Sampieri, Fernández Collado, \& Baptista Lucio, 2014).

A continuación, se hace una descripción de los instrumentos de recolección de datos que se usaran en esta investigación.

La observación directa: al utilizar este instrumento, se pretende analizar las dificultades que se presentan, para desarrollar las diferentes actividades físicas, recreativas y deportivas, no solo en las clases de educación física por parte del docente, sino también en el tiempo libre de los estudiantes. Esta información será confiable ya que el investigador estará presente durante las clases realizando la observación y de acuerdo con las dificultades de análisis que se presenten y las estrategias que se tienen para solucionarlas. Luego de realizar la observación, se analizan los datos que se recogieron y se interpretan en forma cualitativa.

La encuesta consiste en una serie de preguntas de varios tipos, preparadas con base a las dificultades que presenta la investigación, se destaca que, para los cinco grupos escogidos, hay preguntas comunes y otras exclusivas para el grupo de estudiantes y docentes que son los que más se ven perjudicados por el tema de los espacios y escenarios aptos para el desarrollo de las actividades programadas por las áreas del conocimiento. La encuesta tiene como

Volumen 2. Número 2. Julio - Diciembre 2022 
finalidad obtener de manera ordenada información, acerca de las dificultades que tienen los estudiantes y los docentes que utilizan los espacios para la práctica y la realización de las actividades físicas, recreativas, deportivas y culturales (Hernández Sampieri, Fernández Collado, \& Baptista Lucio, 2014).

El interés al usar este instrumento es saber que la comunidad educativa del colegio distrital Carlos Albán Holguín y su opinión, ayudara a desarrollar las dificultades en la solución de los espacios y escenarios que tiene la institución. Se realizó la encuesta con un total de 27 preguntas distribuidas así: diez para los estudiantes, cinco para los docentes, cuatro para los padres de familia, cuatro para los directivos docentes y cuatro para el personal de servicios generales.

\section{Resultados}

Los directivos docentes y administrativos expresan de manera unánime el máximo nivel de importancia frente a la práctica de actividad física-deportiva por parte de sus hijos. Todos los encuestados manifiestan el valor del ejercicio en el desarrollo y bienestar de los hijos, este grupo de encuestados sabe de los beneficios de mantener a sus hijos realizando alguna actividad preferiblemente artística o deportiva en su tiempo libre, para poder mejorar en aspectos que facilitan su desarrollo integral, estas actividades deben ser del gusto de los hijos. De acuerdo con la Carta Internacional de la Educación Física, la Actividad Física y el Deporte 2015, que dice textualmente: "Todo ser humano tiene el derecho fundamental de acceder a la educación física, la actividad física y el deporte sin discriminación alguna, ya esté está basada en criterios étnicos, el sexo, la orientación sexual, el idioma, la religión, la opinión política, o de cualquier otra índole, el origen nacional o social la posición económica o cualquier otro factor" (Unesco, 2013, p.27).

Los padres de familia y el personal de servicios generales consideran muy importante para sus hijos, la práctica de actividades físicas y deportivas. Demostrando el conocimiento que tienen acerca de los beneficios para la salud y la calidad de vida cuando se realizan ejercicios físicos. 
Todas las respuestas a esta pregunta, los padres de los estudiantes estuvieron de acuerdo con que su hijo realice actividades físicas, recreativas y deportivas, para crear hábitos saludables y permanentes en la realización de ejercicios.

A continuación, en el texto de Educación física: derecho a la educación de calidad y bienestar dice: "en este sentido, la actividad física, deportiva y recreativa en la escuela es esencial ligarla a proyectos educativos más integrales como la ética del cuidado del cuerpo" (Pérez, 2016, p.3). Por otra parte, de acuerdo con los estudios realizados por diferentes investigadores las enfermedades crónicas y cardiovasculares se inician en la niñez, debido a muchos factores como el sedentarismo, motivo por el cual los encuestados ven importante el practicar actividades físicas y deportivas desde su niñez (Casimiro Andujar \& Pierón, 1999).

Los docentes ven en la práctica de actividad física, la solución a todas las enfermedades generadas por el sedentarismo tan incrustado en la sociedad moderna, debido a las diferentes ocupaciones y a la falta de buenos espacios para la práctica de actividades físicas. Los docentes que practican actividad física lo hacen por salud, liberar el estrés, por gusto, por bienestar físico, recreativo y por mantener un buen estado emocional, otros practican por diversión, quemar grasa, por mantenimiento, por conservación. Consideran los docentes que si se tienen los implementos necesarios para realizar ejercicios motivaran a aquellos que no realizan actividad física a practicarlas.

A causa de ello en la Carta Internacional de la Educación Física, la Actividad Física y el Deporte 2015, que dice textualmente: "la posibilidad de desarrollar el bienestar y las capacidades físicas, psicológicas y sociales por medio de estas actividades debe verse respaldada por todas las instituciones gubernamentales, deportivas y educativas" (Unesco, 2013, p.27).

El agrado por las actividades físicas por parte de los estudiantes en el colegio es muy importante, debido a la aceptación masiva que tienen en la realización de los ejercicios durante las clases, sin embargo hay una minoría que no gusta de las practicas físicas, recreativas y deportivas, por tal motivo se busca incentivar a esa pequeña minoría para que se una al grupo

Volumen 2. Número 2. Julio - Diciembre 2022 
mayoritario y participe con entusiasmo en la realización de las prácticas preparadas para las clases, esto se lograra teniendo buenos espacios y escenarios, la inclusión de deportes que agraden a este grupo de estudiantes y los implementos necesarios para la práctica. Los estudiantes respondieron que se encuentran altamente satisfechos por las diferentes actividades físicas que se desarrollan durante las clases de educación física.

A esto se añade el artículo de Pierón, Ruiz, \& García (2008), acerca de "La opinión del alumnado de enseñanza secundaria sobre las clases de Educación Física: un desafío para los profesores y formadores" que dice:

El reconocimiento creciente de la actividad física y sus beneficios relacionados con la salud son ejemplo de la necesidad existente de que los educadores físicos, que están adecuadamente preparados para facilitar el desarrollo de las habilidades, los conocimientos, las actitudes y los niveles de "fitness", deben ayudar a sus alumnos a mantener un estilo de vida físicamente activo para siempre. (p.160)

Todavía cabe señalar, con referencia acerca del uso de los espacios en los horarios establecidos ya sean durante las clases o en los eventos deportivos, los estudiantes en su opinión se encuentran parejos, sobre si son suficientes o insuficientes. La mayoría manifiesta que son suficientes, pero no todos los encuestados opinaron lo mismo, por tal motivo necesitan más horas para realizar actividades físicas, deportivas y recreativas, además de incrementar una hora de clase en aquellos grados que no tengan las tres horas de educación física, lo mismo que estos espacios puedan ser utilizados para el aprovechamiento del tiempo libre.

A continuación de acuerdo con la ley 181 de 1995 que dice en el artículo 3: "para garantizar el acceso del individuo y de la comunidad al conocimiento y práctica del deporte, la recreación y el aprovechamiento del tiempo libre, el Estado tendrá en cuenta los siguientes objetivos: 1. Integrar la educación y las actividades físicas, deportivas y recreativas en el sistema educativo general en todos sus niveles" (Congreso de Colombia, 1995, p.1). 
Los docentes expresan en su respuesta, inconformidad por el regular estado en que se encuentran los espacios para la práctica de actividad física en el colegio, deben ser mejorados con todas las normas establecidas de seguridad, demarcación, luz artificial y los implementos. Otro grupo de docentes responde que están en buen estado, también es importante analizar la respuesta de aquellos docentes que reconocen que los espacios se encuentran en mal estado. Sin embargo, con este grupo de docentes se debe generar una correlación con el número de docentes que practican actividad física. Asimismo: "Todos los centros docentes, públicos o privados, deberán disponer de instalaciones deportivas para atender la Educación Física y la práctica del deporte, en las condiciones que se determinen reglamentariamente" (Latorre, 2008, p.63).

Por tal motivo: "Un mayor cumplimiento y seguimiento por parte de las Administraciones correspondientes de la normativa tanto a nivel de construcción inicial como de mantenimiento y/o remodelación de los espacios" (Burillo, Felipe, Gallardo, García, \& Gil, 2010, p.300).

Los estudiantes se refieren a la calidad de los espacios que existen en la institución, con mucho pesimismo ya que manifiestan que son regulares en su gran mayoría, solo un buen grupo manifiestan que estos espacios son buenos para realizar ejercicios. Los estudiantes se encuentran preocupados por el regular estado de los espacios para la realización de las actividades físicas y solicitan el mejoramiento de los mismos para las prácticas que se realizan no solo en educación física sino también en las actividades que desarrollan las demás asignaturas, pero sin embargo existe un grupo que está conforme y afirman que en los espacios que hay se pueden realizar las clases de educación física, la recreación y el deporte sin ninguna dificultad.

Por tanto, le corresponde al estado: "Planificar y programar la construcción de instalaciones deportivas con los equipamientos necesarios, procurando su optima utilización y uso de los equipos y materiales destinados a la práctica del deporte y la recreación” (Congreso de Colombia, 1995, p.2). 
También es importante conocer el estado, en que se encuentran los escenarios deportivos del colegio para la práctica de actividad física, deportiva y la recreación por parte de los padres de familia, que observan con cierto pesimismo el regular estado de dichos escenarios, de los padres encuestados respondieron que estos escenarios se encuentran en regular estado, por tal motivo piden el mejoramiento para evitar accidentes en las clases de educación física y en todas las actividades que se hagan en estos espacios. Las otras respuestas a la pregunta, si están en buen o mal estado estos escenarios se encuentra repartida equitativamente.

Así:

La evaluación de los espacios y equipamientos deportivos escolares es una labor decisiva para prevenir muchos riesgos en la clase de Educación Física; consideramos que es una competencia profesional imprescindible, aunque ante esta no exista gran sensibilización, ni mucho menos instrumentos de análisis operativos y fiables (Latorre, 2008, p.68).

Se observa de acuerdo con el análisis, que para el personal de servicios generales los espacios del colegio son percibidos en un estado óptimo por lo parejo de las respuestas dadas por este grupo de encuestados en el buen y regular estado de estos espacios, solicitan que se deben mejorar estos espacios en cuanto a la demarcación, seguridad e iluminación para realizar actividades físicas y deportivas.

A consecuencia de lo anterior según Tercedor, Jiménez, \& Moya (1994):

En la actualidad se muestra un interés creciente ya no solo hacia la cantidad de instalaciones y disponibilidad de las mismas, sino también hacia la calidad de la instalación y equipamientos. Cuando se trata de fomentar la actividad física orientada hacia la salud, quizá el conocer las posibilidades y calidad de la instalación de que disponemos sea una de las primeras cuestiones a responder. (p.1). 
Los directivos docentes y administrativos en su gran mayoría, expresan que los espacios existentes en el colegio son suficientes; Por otro lado es importante saber que un buen grupo de los encuestados, manifiestan estar satisfechos con los espacios recreo deportivos existentes en la institución, en contraste con la opinión de los otros grupos encuestados que está en desacuerdo con la cantidad de espacios para poder desarrollar actividades físicas, deportivas y recreativas; este grupo solicita la construcción de otros escenarios deportivos o el tener aulas especiales para la práctica de deportes que se realicen en este tipo de escenarios.

Resulta oportuno el aporte del Decreto 4183 de 2011 que dice textualmente: "Fomentar la generación y creación de espacios que faciliten la actividad física, la recreación y el deporte" (p.3).

Los padres de familia y el personal de servicios generales ven con pesimismo la cantidad de espacios deportivos, que tiene la institución para la práctica de actividad física, los deportes y la recreación por parte de sus hijos, ya que de acuerdo con las respuestas dadas consideran insuficientes los espacios deportivos. Los padres encuestados consideran que existe hacinamiento, ya que la institución no tiene los suficientes espacios para el desarrollo de las clases de educación física y las actividades deportivas y recreativas que deben realizar sus hijos, perjudicando el desarrollo integral y la creación de hábitos en la práctica de ejercicios, sin embargo, una minoría considera que con los espacios que hay son suficientes para desarrollar dichas actividades.

Con referencia a lo anterior es importante anotar: "Formular, adoptar, coordinar la ejecución y evaluar estrategias de la promoción, el fomento, el desarrollo y la orientación del deporte, la recreación, la actividad física y el aprovechamiento del tiempo libre" (República de Colombia, 2011, p.2). Es evidente entonces tener en cuenta: "Planificar y programar la construcción de instalaciones deportivas con los equipamientos necesarios, procurando su óptima utilización y uso de los equipos y materiales destinados a la práctica del deporte y la recreación” (República de Colombia, 2011, p.3). 
Hay diferentes puntos de vista, frente a la construcción de nuevos escenarios, en este sentido se aprecia que el tenis de mesa, el voleibol, el gimnasio y el futbol cinco, son algunos de los espacios que los directivos docentes y administrativos consideran se deben construir como prioridad, asimismo deportes como el baloncesto, tenis de campo, máquinas para realizar ejercicios, billar deben ser considerados para que si hay los espacios se puedan construir estos escenarios deportivos.

Los docentes en sus respuestas consideran la importancia de construir nuevos y buenos espacios, para que la comunidad educativa se motive a practicar actividades físicas, deportivas y recreativas, en estos espacios nuevos. Un gimnasio bien dotado, un coliseo adecuado, un salón de danzas; dos canchas múltiples, un salón de expresión corporal, un salón de pesas, canchas cubiertas, una zona de entrenamiento, espacios para baloncesto, futbol cinco y voleibol, una pista de patinaje, una pista de atletismo, en ese orden de preferencia los docentes piden que se construyan estos escenarios, además no hay interés para que se construya una cancha de tenis de campo.

Con referencia a lo anterior: "En muchos casos la limitación de recursos determina la ejecución de la construcción escolar por fases que serán determinadas y priorizadas de acuerdo con las expectativas y posibilidades institucionales" (Benavides, 2006, p.157).

Los estudiantes de la institución anhelan tener buenos espacios para la práctica de actividades físicas y deportivas, teniendo en cuenta las respuestas dadas a la adecuación de escenarios deportivos, desean que estos sean bastantes y variados para la participación de toda la comunidad educativa, mejorando la calidad de vida. Los estudiantes tienen por gusto la gimnasia, por tal motivo solicitan la construcción de un gimnasio, con todos los elementos necesarios para la práctica de la misma, también desean la construcción de los siguientes escenarios: canchas de baloncesto, fútbol cinco, banquitas, espacio para tenis de mesa, canchas de voleibol, lo mismo de tener espacios aptos para el ajedrez y el billar, canchas de tenis de campo; otro deseo es que el deporte autóctono el tejo se considere. Teniendo en cuenta que en nuestro colegio el fútbol es importante un buen grupo anhela una cancha de fútbol. 
Estos dos últimos deportes es imposible su construcción debido a las dimensiones que tienen ya que en el colegio no existe tanto espacio para la construcción de estos escenarios (Benavides, 2006).

Los padres de familia ven con optimismo en la nueva construcción y remodelación del colegio la posibilidad de tener nuevos escenarios, por tal motivo solicitan escenarios deportivos aptos y de calidad para las actividades físicas, deportivas y recreativas, por consiguiente, desean que sus hijos las practiquen, no solo durante las clases de educación física sino también en el tiempo libre. Un gimnasio, una cancha de fútbol, escenarios de futbol cinco, baloncesto, voleibol, un salón de máquinas de ejercicio, una pista de patinaje, espacios para tenis de mesa, billar y tenis de campo, son los escenarios que los padres de familia o acudientes de los estudiantes desean que se construyan en la institución en ese mismo orden de opinión.

Por tal motivo al tener todos estos escenarios, la importancia de la actividad física en la institución será relevante y puede llamar la atención a los habitantes del sector, para que matriculen a sus hijos en el colegio. En efecto: "el material didáctico los equipos y los currículos flexibles y accesibles pueden ser clave para crear escuelas inclusivas" (Unesco, 2015, p.32).

Existen diferentes opiniones acerca de la construcción de los nuevos escenarios deportivos, por parte del personal de servicios generales, el baloncesto, el voleibol, el futbol cinco, un gimnasio, son de buena aceptación y son vistos por este grupo de encuestados como las principales opciones para que el colegio los asuma en la nueva planta física que tendrá la institución, además prefieren la construcción de espacios para el tenis de mesa, billar, tenis de campo, máquinas de ejercicio, una pista de patinaje y una cancha para la práctica del fútbol. Por otro lado, este grupo de encuestados considera que con buenos escenarios los estudiantes participarán activamente en las clases y asistirán con entusiasmo a las actividades deportivas que se programan en el tiempo libre. 
Es evidente entonces que: "Fomentar la creación de espacios que faciliten la actividad física, el deporte y la recreación como habito de salud y mejoramiento de la calidad de vida y el bienestar social" (Congreso de Colombia, 1995, p.1).

La respuesta a esta pregunta por parte de los docentes es contundente, todas las personas encuestadas, manifestaron la necesidad de tener buenos espacios, para la práctica de actividad física en el colegio. Además, los docentes piden que estos espacios sean seguros, suficientes, con buena demarcación, iluminación y que tengan los implementos para la práctica de las diferentes disciplinas deportivas. Asimismo, estos espacios deben servir para las actividades que se realizan no solo en educación física sino también en las otras áreas del conocimiento. De acuerdo con Latorre (2008) cuando escribe:

La organización de los espacios deportivos va a estar determinada por la dotación del centro escolar y por la realización de actividades simultáneas y compartidas con otros docentes. La escasez de instalaciones es un condicionante que estimula la creatividad del profesorado y en el peor de los casos puede comprometer la seguridad. (p.64).

\section{Discusión}

Por parte de la comunidad educativa se considera importante que los jóvenes realicen actividades físicas, deportivas o recreativas para que mejoren su parte física, la salud y se mantengan ocupados en actividades positivas, creando hábitos saludables de vida. Como lo dice en Alfaro, Campos, Holst, \& Núñez, (2015): "Promover en los niños y jóvenes la actividad física en juegos, deportes, desplazamientos, actividades recreativas, educación física o ejercicios programados" (p.375).

La comunidad educativa en relación a la pregunta sobre el estado de los espacios que posee el colegio se encuentra insatisfecha, ya que lo escenarios para la práctica de la actividad física son muy regulares. En el artículo de Benítez \& Sequera (2009) nos dice:

Volumen 2. Número 2. Julio - Diciembre 2022 
Debido a lo expuesto con anterioridad, se hace necesario el diseño de una estrategia que permita mejorar el mantenimiento en dichos espacios, teniendo como elementos principales la organización estructural, la gestión de actividades físicos-deportivasrecreativas y la capacitación del personal encargado de mantener las instalaciones. (p.1)

La percepción sobre los espacios deportivos en la institución está dividida en opinión dada por los directivos docentes, padres de familia y el personal de servicios generales, ya que un buen grupo manifiesta estar inconforme con la cantidad de escenarios existentes en la institución, mientras que otro grupo, opina que con los espacios que cuenta el colegio son suficientes, para realizar actividades físicas, recreativas y deportivas, lo mismo que para aprovecharlos en las actividades que se puedan realizar en el tiempo libre.

El objetivo principal que se busca en esta investigación es contar con la cantidad y calidad de instalaciones, suficientes para cada docente del área de educación física, para así poder desarrollar las prácticas pedagógicas de actividad física y las recreativas (Burillo, Felipe, Gallardo, García, \& Gil, 2010).

La comunidad educativa, expreso su opinión acerca de la construcción de escenarios para la práctica de actividad física, deportiva y recreativa, en la remodelación del colegio, donde desean que la institución tenga, como prioridad un gimnasio bien dotado, lo mismo que un espacio cerrado para voleibol y tenis de mesa, los demás deportes como el baloncesto, fútbol cinco, billar, el fútbol, tenis de campo, pista de patinaje, máquinas de ejercicio, también tienen acogida dentro de las opiniones de la comunidad educativa para que sean estudiados y si existe la posibilidad de que sean construidos, se hagan de acuerdo con las dimensiones reglamentarias y que se construyan, con la prioridad de la opinión facilitada por los integrantes del grupo encuestado.

De acuerdo con Benavides, (2006): 
Esta diferenciación debe evidenciarse en los ambientes y áreas que se incorporan a la escuela; el tamaño y proporción de estas áreas también debe ser variada, desde los pequeños lugares de encuentro, hasta las grandes plazas y patios de banderas donde coincide toda la comunidad educativa. Desde el ruidoso y "efervescente" patio de recreo, hasta las tranquilas zonas verdes que se convierten en "playas" de descanso, las áreas recreativas son ambientes fundamentales del hábitat escolar (p.182).

Se analizó los resultados de la encuesta a los directivos docentes, docentes, estudiantes, padres de familia y personal de servicios generales, se consolido la información registrada y se presentan las siguientes observaciones:

El conjunto de evidencias, manifiesta la importancia que tiene la práctica de actividad física para la comunidad educativa, realizada por parte de sus hijos, asimismo se acepta que las actividades físicas son vitales para mejorar la salud y la calidad de vida, además los estudiantes solicitan incluir en las clases de educación física nuevas actividades deportivas y manifiestan que los ejercicios realizados en las clases de educación física son buenos y adecuados para su formación integral, también solicitan aumentar una hora de clase en todos los grados que solamente tengan 2 horas semanales, lo mismo que los espacios sean prestados los días que no se tenga clase para realizar actividades físicas en su tiempo libre.

De acuerdo con las respuestas dadas, los espacios en el colegio se encuentran en regular estado para la práctica de actividad física y deportiva, opinión contundente de la comunidad educativa, por otro lado según la encuesta, la opinión sobre la cantidad de escenarios que hay en la institución está dividida, ya que un grupo de los encuestados dice que son suficientes y el otro grupo manifiesta que hacen faltas escenarios para las actividades físicas, además existen diferentes opiniones, para la construcción de escenarios en la remodelación del colegio, pero el deseo general es que sean varios y ojalá teniendo en cuenta los resultados de la encuesta. 
En cuanto al aprovechamiento del tiempo libre, las opiniones a las diferentes preguntas realizadas son variadas, ya que los encuestados opinan que los espacios que existen deben mejorar y que no son suficientes para la cantidad de estudiantes que existen en el colegio, además la mayoría de estudiantes realizan diferentes actividades deportivas, en escuelas deportivas o por sí mismo, y la opinión acerca de los eventos que se realizan en la institución como aprovechamiento del tiempo libre, son suficientes por la variedad de los mismos y la competencia que existe entre los diferentes equipos de los grados participantes.

\section{Conclusiones}

Como resultado, se identificó la necesidad de tener buenos y varios espacios adecuados para la práctica de la recreación y el deporte, esta condición puede ser importante para el desarrollo de las actividades propuestas y así lograr la participación de la gran mayoría de los estudiantes. De manera que, si se mejoran los escenarios y espacios para la realización de las clases de educación física, con muy buenos implementos y teniendo seguridad en los ambientes para la práctica, se pueden alcanzar buenos niveles en la calidad de la educación impartida en la institución.

Por tanto, para la comunidad educativa, es importante la práctica de actividad física por parte de los hijos, ya que, si esta se realiza frecuentemente, ayuda al desarrollo y bienestar, beneficios para la salud y la calidad de vida, y así crear hábitos de ejercicios por parte de los hijos.

En relación con las actividades físicas en el colegio para los estudiantes, manifiestan que son importantes y están satisfechos con las diferentes prácticas que se hacen en las clases de educación física, sin embargo, existe una minoría que no le gusta, ni practica ningún tipo de ejercicios, con ellos se debe buscar mecanismos para incentivarlos en la práctica de actividad física y deportiva. 
En la actualidad el colegio se encuentra en lista para su remodelación, de una buena parte de sus instalaciones físicas, lo mismo que de los espacios deportivos, por tal motivo la comunidad educativa, solicita la construcción de los siguientes escenarios, considerando los posibles espacios que se podrán utilizar para tal objetivo: voleibol, baloncesto, fútbol cinco ojalá que estos escenarios sean encerrados, aulas para el tenis de mesa, salón de danzas y un gimnasio bien dotado.

La falta de escenarios deportivos es percibida en la institución, por tal motivo los estudiantes piden la construcción de más escenarios, para poder desarrollar las actividades físicas en las clases y realizarlas en el tiempo libre. Este es un aspecto para considerar por los entes territoriales.

Los eventos deportivos y recreativos, los diferentes torneos que realiza el colegio por medio del área de educación física son suficientes, y motivan a su participación voluntaria y masiva por parte de los estudiantes, puesto que se juega no solo en horas de descanso, sino también sábados y festivos, además los estímulos que se otorgan a los ganadores por parte de rectoría son llamativos y así se puede cumplir con el proyecto aprovechamiento del tiempo libre.

\section{Referencias}

Alfaro, V., Campos, N., Holst, I., \& Núñez, P. (2015). De las creencias de la comunidad educativa escolar sobre Actividad Física a las políticas institucionalesdel centro educativo. Revista de currículum y formación del profesorado, 373-388.

Benavides, C. (2006). Hábitat escolar más allá de la infraestructura educativa. Bogotá D.C: Escala.

Benítez, J., \& Sequera, D. (2009). El mantenimiento de los espacios para la actividad física deportiva en la UniversidadIbeoamericana del deporte. efdeportes.com, 1-4. 
Bull, F., Jacoby, E., \& Neiman, A. (2003). Cambios acelerados del estilo de vida obligan a fomentar la actividad física como prioridad en la Región de las Américas. Panamericana de Salud Pública, 1-3.

Burillo, P., Felipe, J., Gallardo, L., García, M., \& Gil, J. (2010). Detección de necesidades en las instalaciones deportivas de educación secundaria obligatoria: El caso de la provincia de Ávila. Journal of sport and health research, 287-304.

Casimiro Andujar, A. J., \& Pierón, M. (1999). Incidencia de la práctica fíisico-deportiva de los padres hacia sus hijos durante la infancia y la adolescencia. Miscelánea, 100-104.

Colegio Carlos Albán Holguín. (2018). Agenda Escolar. Bogotá D.C., Cundinamarca, Colombia: Alternativa Gráfica Ltda.

Congreso de Colombia. (18 de Enero de 1995). Ley 181. Fomento del deporte, la recreación, el aprovechamiento del tiempo libre y la Educación Física. Bogotá D.C, Colombia.

Galvis, F. (2014). EscenariosDeportivos/Federico Galvis's Blog.

Latorre, P. (2008). Metodología para el analisis y evaluación de la seguridad de los espacios y equipamientos deportivos escolares. Apunts Educación Física Y Deportes, 62-70.

Mollá, M. (2007). La influencia de las actividades extraescolares en los hábitos deportivos de los escolares. Revista Internacional de Medicina y Ciencias de la Actividad Física y el Deporte., 7(27), 241-252.

Nuviala, A., Ruiz, F., \& García, E. (2003). Tiempo libre, ocio y actividad física en los adolescentes. La influencia de los padres. Retos. Nuevas tendencias en Educación Física, Deporte y Recreación., 13-20. 
Pérez, A. (2016). Educación Física. derecho a la educación de calidad y bienestar. Renovación Magisterial, 2-3.

Pierón, M., Ruiz, F., \& García, E. (2008). La opinión del alumnado de enseñanza secundaria sobre las clases de educación física: un desafío para los profesores y los formadores. Fuentes. Volumen 8, 159-175.

República de Colombia. (2011). Decreto 4183 de Noviembre 3 de 2011. 108-125.

Tercedor, P., Jiménez, M., \& Moya, Y. (1994). Los Espacios y Materiales Físico-Deportivos. Conoce sus posibilidades. 1-15.

Ucha, F. (2008). Definición ABC., 15.

Unesco. (30 de Mayo de 2013). Declaración de Berlín. Carta Internacional de la Educación Física, la Actividad Física y el Deporte 2015, 3-5. Berlín, Alemania.

Unesco. (2015). Educación Física de Calidad, guía para los responsables políticos. Reino Unido: Unesco.

Unesco Declaración de Berlín. (2015). Carta Internacional de la Educación Física, la Actividad Física y el Deporte. República de Colombia. 